\title{
Predictive Value of Duration and Frequency of Macroscopic Haematuria for Stage, Prognosis and Recurrence in Bladder Cancer Patients
}

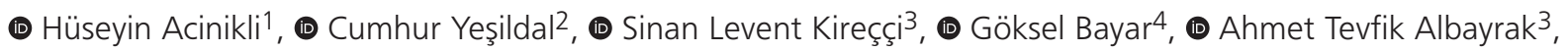 \\ (1) Elsad Abdullayev ${ }^{3}$, (1) Abdullah Hızır Yavuzsan ${ }^{3}$ \\ ${ }^{1}$ Mersin City Hospital, Clinic of Urology, Mersin, Turkey \\ 2University of Health Sciences Turkey, Sultan 2. Abdülhamid Han Training and Research Hospital, Clinic of Urology, Istanbul, Turkey \\ 3 University of Health Sciences Turkey, Şişli Hamidiye Etfal Training and Research Hospital, Clinic of Urology, Istanbul, Turkey \\ ${ }^{4}$ Sancaktepe Şehit Prof. Dr. Ilhan Varank Training and Research Hospital, Clinic of Urology, Istanbul, Turkey
}

\begin{abstract}
Objective: We aimed to investigate the predictive value of duration and frequency of macroscopic haematuria for the stage, prognosis and recurrence rates in primary bladder cancer.

Materials and Methods: We retrospectively reviewed the data of our patients diagnosed with primary bladder cancers during 2000-2014. Patients with history of macroscopic haematuria were included. Their haematuria duration and frequency and stage and grade of bladder cancer, recurrence rates, time until recurrence, time until progression and pre-operative use of anticoagulants were evaluated.

Results: A total of 331 patients comprising 276 males (83\%) and 55 females (16\%) were included in the study. The mean age of the patients was $64.0 \pm 11.8$ (28-93) years. The average haematuria duration was 18.5 \pm 33.5 (0-260) months. There were $173(52 \%), 106(32 \%)$ and $52(15.7 \%)$ patients with cancer stages of Ta, T1 and T2, respectively. The average follow-up time was 54.0 $\pm 41.8(1-268)$ months. The frequency of haematuria was significantly higher in patients with muscle-invasive bladder cancers than in those with superficial bladder cancers $(p=0.010)$. Similarly, patients with tumour diameter $>3 \mathrm{~cm}$ reported significantly higher frequency of haematuria than in those with tumour diameter $<3 \mathrm{~cm}(p=0.045)$. Five patients exclude from study because they did not attend their follow-up. During follow-up recurrences were seen in 89 (32.3\%) out of 326 patients, while 237 (72.7\%) patients did have any recurrences. Disease progression was reported in 28 (8.5\%) patients. The average time periods until recurrence and progression were $25.8 \pm 34.7$ months and $27.1 \pm 34.9$ (1-144) months, respectively.

Conclusion: We found a significant difference between the frequency of haematuria in patients with muscle-invasive cancers and those with superficial cancers. Also, we found a negative correlation between smoking and the time until recurrence in patients with macroscopic haematuria. No significant relationships were observed between the duration of haematuria and cancer recurrence rates and prognosis.

Keywords: Haematuria, bladder cancer, haematuria frequency, haematuria duration
\end{abstract}

\section{Introduction}

Bladder cancers are one of the most common cancers of the urinary system and comprise $2 \%-3 \%$ and $6 \%-8 \%$ of malignant cancers in women and men, respectively $(1,2)$. Owing to their low prevalence in the general population, their screening is not recommended (3). Factors affecting their prognoses are tumour burden, diameter, composition, histology, stage, grade and accompanying carcinoma in situ (CIS) (4). Among the various diagnostic tools, cystoscopy is the best modality available for urologists to diagnose bladder cancers (5). The most common symptom of bladder cancer is painless macroscopic haematuria. It is seen in $85 \%$ of bladder cancer patients. Microscopic haematuria can be found in almost all patients. The second most common symptom comprises irritable bladder symptoms that are seen in $20 \%$ of patients. These indicate the presence of CIS or invasive bladder cancer (6). Haematuria is commonly seen in the general population, with a prevalence of $2.5 \%-20 \%$ $(7,8)$. The diagnosis and degree of haematuria can be found by counting red blood cells in each $1 \mathrm{~mL}$ of urine sample (chamber count), via sediment count, or through an indirect dipstick test (9). An underlying urinary tract cancer is present in $40 \%$ and

Cite this article as: Acinikli H, Yeşildal C, Kireççi SL, Bayar G, Albayrak AT, Abdullayev E, Yavuzsan AH. Predictive Value of Duration and Frequency of Macroscopic Haematuria for Stage, Prognosis and Recurrence in Bladder Cancer Patients. Bull Urooncol 2021;20(1):34-39

Address for Correspondence: Cumhur Yeşildal, University of Health Sciences Turkey, Sultan 2. Abdülhamid Han Training and Research Hospital, Clinic of Urology, İstanbul, Turkey Phone: +90 5336815658 E-mail: c_yesildal@hotmail.com ORCID-ID: orcid.org/0000-0002-1518-8371 
approximately $5 \%$ of patients with macroscopic and microscopic haematuria, respectively (10). No underlying illness is seen in $40 \%$ of patients with microscopic haematuria (7). Haematuria may not always be a cause for alarm; studies have shown that $9 \%-18 \%$ of healthy individuals may report this symptom (11).

Many studies have showcased the importance of microscopic haematuria; unfortunately, there are limited publications investigating macroscopic haematuria. Some studies have explored the association between macroscopic hematuria and bladder cancer.

However, no previous study has explored the relationship between the frequency and duration of haematuria with the stage, degree, recurrence, prognosis, tumour burden, diameter, cigarette use, age, body mass index (BMI) and pre-operative anticoagulant usage. We aimed to determine the effect of the frequency and duration of macroscopic haematuria on primary bladder cancers; primarily with the stage, prognosis and recurrence rate.

\section{Materials and Methods}

The study was approved by the ethics committee of our hospital, the Şişli Hamidiye Etfal Training and Research Hospital. All patients included in the study were informed about the study and written consents were obtained. Data of the patients with primary bladder cancers attending the urology clinic at the hospital during 2000-2014 were retrospectively evaluated. A total of 331 patients were included in the study and divided into two groups based on the presence and absence of macroscopic haematuria. Haematuria duration and frequency, tumour characteristics namely; stage, grade, recurrences, time until recurrence, time until progression and pre-operative anticoagulant usage were reviewed.

Furthermore, patients were tested for CIS. Then the tumour burden and intravesical therapies [Bacillus Calmette-Guerin (BCG) or chemotherapy] were noted. Patients with macroscopic haematuria were divided into four groups, based on prior studies, considering the duration of the symptom as: group 1: less than 3 months, group 2: 3-5 months, group 3: 6-12 months and group 4: longer than 12 months. Duration of haematuria was calculated from the time of its first appearance until the date of operation, while its frequency was defined as the number of days on which haematuria occurred. Pre-operative $\mathrm{Hb}$ and $\mathrm{Htc}$ values were noted for all patients. Patients were followed up for 5 years. Their controls were planned as per the EAU guidelines.

\section{Statistical Analysis}

SPSS 15.0 for Windows was used for the statistical analysis. Descriptive statistics were employed for the evaluation of results: for categorical variables, numbers and percentages and for numerical variables, mean, standard deviation, minimum, maximum and median values were used. The Mann-Whitney $U$ test was used for continuous variables. The ratio of the categorical variables between the groups was tested by chi-square analysis. A p-value $<0.05$ was considered as statistically significant.

\section{Results}

A total of 331 patients were included in our study. Demographic data of the patients are listed in Table 1. There were 276 (83\%) men and 55 (16\%) women. Data are presented as mean \pm standart deviation. The average age was $64.0 \pm 11.8$ (28-93) years and the average BMI was $26.7 \pm 4.2(16-39) \mathrm{kg} / \mathrm{m}^{2}$. Mean cigarette usage per year was $33.8 \pm 28.5$ (0-150) packs/year. Sixty-one patients (21.9\%) showed no signs of macroscopic haematuria, while 218 patients $(78.1 \%)$ did. The mean haematuria duration was $18.5 \pm 33.5(0-260)$ months. Patients with haematuria were divided into four groups according to their symptom duration as mentioned earlier. Groups I-IV had 121 (43.4\%), 35 (12.5\%), 15 (5.4\%) and 47 (16.8\%) patients, respectively. Frequency of haematuria was $10.4 \pm 31.9$ (0-365) days. Pre-op $\mathrm{Hb}$ values were $13.5 \pm 2.1 \mathrm{gm} / \mathrm{dL}$ and pre-op Hct values were $40.7 \pm 6.0$ (13.7-51.8)\%. Pathological staging of $\mathrm{Ta}$, $\mathrm{T} 1$, and T2 bladder cancers were seen in 173 (52\%), 106 (32\%) and $52(15.7 \%)$ patients.

The average follow-up time was $54.0 \pm 41.8$ months. There were $40(30.5 \%)$ and 91 (69.5\%) patients with tumours smaller and larger than $3 \mathrm{~cm}$, respectively. Regarding tumour focality, 92 patients $(66.7 \%)$ had unifocal, $36(26.1 \%)$ had $2-7$ foci and 10 (7.2\%) patients had bulky tumours.

\begin{tabular}{|c|c|c|}
\hline Age & & $64.0 \pm 11.8 / 28-93$ \\
\hline \multirow{2}{*}{ Sex } & Male & $276(83.4)$ \\
\hline & Female & $55(16.6)$ \\
\hline Height & & $168.7 \pm 7.7 / 150-190$ \\
\hline Weight (kg) & & $75.8 \pm 12.2 / 48-113$ \\
\hline BMI & & $26.7 \pm 4.2 / 16-39.0$ \\
\hline Cigarettes (packs/year) & & $33.8 \pm 28.5 / 0-150$ \\
\hline \multirow{6}{*}{ Comorbid Illness } & HT & $117(36.7)$ \\
\hline & DM & $61(19.1)$ \\
\hline & IHD & $40(12.5)$ \\
\hline & CBF & $9(2.8)$ \\
\hline & COPD & $26(8.2)$ \\
\hline & Other & $10(3.1)$ \\
\hline Hematuria interval & & $18.5 \pm 33.5 / 0-260$ \\
\hline \multirow{6}{*}{ Hematuria interval } & Negative & $61(21.9)$ \\
\hline & Positive & $218(78.1)$ \\
\hline & $<3$ months & $121(43.4)$ \\
\hline & 3-5 months & $35(12.5)$ \\
\hline & 6-12 months & $15(5.4)$ \\
\hline & $>12$ months & $47(16.8)$ \\
\hline Days with hematuria & & $10.4 \pm 31.9 / 0-365$ \\
\hline Pre-op Hb & & $13.5 \pm 2.1 / 6.1-17.8$ \\
\hline Pre-op HCT & & $40.7 \pm 6.0 / 13.7-51.8$ \\
\hline
\end{tabular}

BMI: Body mass index, Hb: Haemoglobin, HCT: Hematocrit, HT: Hypertension, DM: Diabetes mellitus, IHD: Ischaemic heart disease, CBF: Chronic bladder failure, COPD: Chronic obstructive pulmonary disease 
Recurrences were observed in 89 out of 326 patients (27.3\%) during their follow-up; while 237 patients (72.7\%) remained in remission. Disease progression was observed in 28 (8.5\%) patients. Average time periods until recurrence and progression were $25.8 \pm 34.7$ and $27.1 \pm 34.9$ (1-144) months, respectively. Furthermore, while 46 (15.8\%) patients underwent intracavitary chemotherapy, 63 (21.6\%) patients received intracavitary BCG. Anticoagulants were received by 27 patients pre-operatively, while 174 (86.6\%) did not receive any (Table 2 ).

On statistical analyses, the relation between age and haematuria duration was negatively significant $(p=0.024)$. The average haematuria duration was significantly higher in men than in women $(p=0.035)$.

Tumours larger than $3 \mathrm{~cm}$ in diameter were found to be significantly more in patients with haematuria than in those without it $(p<0.001)$.

No meaningful differences were determined between tumour burden and haematuria duration. The same was true for frequency of haematuria, recurrence ratio, time until recurrence, number of recurrences, progression rate and time until progression.

Frequency of haematuria was significantly higher in patients with a tumour diameter $>3 \mathrm{~cm}$ than in those with tumour diameter $<3 \mathrm{~cm}(\mathrm{p}=0.045)$. However, such an association was not seen with haematuria duration. Also, frequency of haematuria, recurrence rate, time until recurrence, the number of recurrences, progression rate and time until progression are not significantly associated with haematuria duration.

Frequency of haematuria was significantly associated with the pathological staging of the tumour $(p=0.022)$. Stage Ta patients had a significantly lower frequency compared with that of stage T2 patients $(p=0.021)$. Haematuria duration, recurrence rate, time until recurrence, progression rate and time until progression were not significantly correlated with the pathological staging. The grade of tumours were found to be significantly different among the groups based on the haematuria duration $(p=0.033)$. In patients with grade 1 tumours, the ratio of those with a haematuria interval of 6-12 months was determined to be highest. There was no significant difference in frequency of haematuria, recurrence rate, recurrence quantity and progression rate and time until progression among the different grades.

Superficial bladder cancers were further divided into three groups: low, intermediate and high risk; and their characteristics were analysed. No significant differences were found among the groups in terms of haematuria duration, frequency, recurrence rate, time until recurrence, number of recurrences and progression rate and time until progression.

A positively significant correlation was observed between haematuria duration and cigarette usage (packs/year; $p=0.016$ ), while a negatively significant correlation was found between recurrences and cigarette usage (packs/year; $p=0.024$ ). Age and $\mathrm{BMI}$ were shown to have no significant effect on haematuria duration and frequency, number of recurrences, time until recurrence, number of recurrence, and time until progression.
Haematuria duration was not found to be significantly correlated with recurrence rates, progression rates, time until recurrence and time until progression. The same was true for pre-operative anticoagulant use.

In patients without haematuria, no significant differences were observed with regards to burden, tumour diameter, stage, grade, recurrence and progression rates.

In patients with superficial bladder cancers without haematuria, no significant differences were observed in low, intermediate and high-risk groups (Table 3).

Frequency of haematuria was observed to be more statistically significant in muscle-invasive bladder cancer patients than in those with superficial tumours $(p=0.010)$. The tumour burden in patients with superficial tumours was significantly higher than in those with muscle-invasive tumours $(p=0.020)$.

\begin{tabular}{|c|c|c|}
\hline \multirow{3}{*}{ Stage } & $\mathrm{Ta}$ & $173(52.3)$ \\
\hline & T1 & $106(32.0)$ \\
\hline & $\mathrm{T} 2$ & $52(15.7)$ \\
\hline \multirow{3}{*}{ Superficial (non-T2) risk } & Low risk & $119(36.0)$ \\
\hline & Intermediate risk & $34(10.3)$ \\
\hline & High risk & $127(38.1)$ \\
\hline \multirow{3}{*}{ Grade } & 1 & $19(5.8)$ \\
\hline & 2 & $163(50.0)$ \\
\hline & 3 & $144(44.2)$ \\
\hline \multirow{2}{*}{ CIS } & Negative & $278(86.6)$ \\
\hline & Positive & $43(13.4)$ \\
\hline \multirow{2}{*}{ RE-TUR } & Negative & $184(65.2)$ \\
\hline & Positive & $98(34.8)$ \\
\hline \multirow{2}{*}{ Recurrence } & Negative & $237(72.7)$ \\
\hline & Positive & $89(27.3)$ \\
\hline Time until recurrence (months) & & $25.8 \pm 34.7 / 1-206$ \\
\hline \multirow{2}{*}{ Progression } & Negative & $303(91.5)$ \\
\hline & Positive & $28(8.5)$ \\
\hline Time to progression (months) & & $27.1 \pm 34.9 / 1-144$ \\
\hline Follow-up period & & $54.0 \pm 41.8 / 1-268$ \\
\hline \multirow{4}{*}{ Intracavitary treatment } & Negative & $136(46.6)$ \\
\hline & CT & $46(15.8)$ \\
\hline & $\mathrm{BCG}$ & $63(21.6)$ \\
\hline & $\mathrm{CT}+\mathrm{BCG}$ & $47(16.1)$ \\
\hline \multirow{2}{*}{ Pre-op anticoagulant use } & Negative & $174(86.6)$ \\
\hline & Positive & $27(13.4)$ \\
\hline \multirow{3}{*}{ Tumour count } & 1 & $92(66.7)$ \\
\hline & $2-7$ & $36(26.1)$ \\
\hline & $>7$ & $10(7.2)$ \\
\hline \multirow{2}{*}{ Tumour diameter } & $<3 \mathrm{~cm}$ & $40(30.5)$ \\
\hline & $>3 \mathrm{~cm}$ & $91(69.5)$ \\
\hline
\end{tabular}




\begin{tabular}{|c|c|c|c|c|}
\hline & & \multicolumn{2}{|l|}{ Stage } & \multirow[b]{2}{*}{$\mathbf{p}$} \\
\hline & & Superficial & $\mathrm{T} 2$ & \\
\hline Hematuria interval mean \pm SD & & $18 \pm 33.6$ & $21.1 \pm 33.4$ & 0.055 \\
\hline \multirow{5}{*}{ Hematuria interval n (\%) } & Negative & $55(23.8)$ & $6(12.5)$ & 0.254 \\
\hline & $<3$ months & $98(42.4)$ & $23(47.9)$ & \\
\hline & 3-5 months & $29(12.6)$ & $6(12.5)$ & \\
\hline & 6-12 months & $10(4.3)$ & $5(10.4)$ & \\
\hline & $>12$ months & $39(16.9)$ & $8(16.7)$ & \\
\hline Days with hematuria mean \pm SD & & $9.6 \pm 33.3$ & $14.5 \pm 23.3$ & 0.010 \\
\hline \multirow{2}{*}{ Tumour diameter $\mathbf{n}(\%)$} & $<3 \mathrm{~cm}$ & $34(33.0)$ & $6(21.4)$ & 0.238 \\
\hline & $>3 \mathrm{~cm}$ & $69(67.0)$ & $22(78.6)$ & \\
\hline \multirow{3}{*}{ Tumour count $\mathbf{n}(\%)$} & 1 & $68(61.8)$ & $24(85.7)$ & 0.020 \\
\hline & $2-7$ & $34(30.9)$ & $2(7.1)$ & \\
\hline & $>7$ & $8(7.3)$ & $2(7.1)$ & \\
\hline \multirow{2}{*}{ Recurrence $\mathbf{n}(\%)$} & Negative & $192(70.1)$ & $45(86.5)$ & 0.015 \\
\hline & Positive & $82(29.9)$ & $7(13.5)$ & \\
\hline Time till recurrence (ay) mean \pm SD & & $27.1 \pm 35.9$ & $11.4 \pm 7.1$ & 0.250 \\
\hline Recurrence quantity mean \pm SD & & $0.7 \pm 1.5$ & $0.3 \pm 1.0$ & 0.020 \\
\hline \multirow{2}{*}{ Progression n (\%) } & Negative & $254(91.0)$ & $49(94.2)$ & 0.593 \\
\hline & Positive & $25(9.0)$ & $3(5.8)$ & \\
\hline Time till progression (months) mean $\pm S D$ & & $28.7 \pm 36.5$ & $13.3 \pm 9.7$ & 0.710 \\
\hline
\end{tabular}

Patients with superficial bladder cancers showed significantly higher recurrent tumours $(p=0.020)$ and recurrence rates $(p=0.015)$ compared with those in patients with muscle-invasive bladder cancers.

\section{Discussion}

Haematuria is one of the most common symptoms of bladder cancer with majority of bladder cancer patients presenting with macroscopic haematuria. Upon detailed inquiry it has been determined that it is not taken seriously and that most patients seek medical care only after suffering recurrent episodes of haematuria.

Although, microscopic haematuria has been studied in detail, there are limited studies about macroscopic haematuria. Edwards et al. (12) reported that $82.4 \%$ of patients presenting with macroscopic haematuria were diagnosed with bladder cancers. Gandrup et al. (13) studied 150 patients with macroscopic haematuria and reported bladder cancers in 30 patients. Another study stated that if each patient presenting with macroscopic haematuria received a complete urological examination; approximately $10 \%$ will be diagnosed with bladder cancers (14).

The examples above are from studies in which bladder cancer is determined cumulatively from the presence of macroscopic haematuria. However, unlike our study, the association between the duration and frequency of macroscopic haematuria with the stage, grade, recurrence, prognosis, tumour count and diameter, cigarette use, age, BMI and anticoagulant use has not been examined. We aimed to determine the predictive value of the duration and frequency of macroscopic haematuria for the stage, prognosis and recurrence of bladder cancer.

Delayed diagnosis and treatment of bladder cancer can affect survival (15). Further, patients who ignore preliminary symptoms and undergo treatment later have higher tumour grades and decreased survival rates (16). It has also been determined that delays in diagnosis increase the mortality risk related to the stage and grade of the disease (17). Superficial (non-muscle invasive) tumours comprise $60 \%-80 \%$ of the newly diagnosed bladder cancers and majority of them are low grade. Our study detected a ratio of $84.3 \%$ superficial bladder cancers and $15.7 \%$ muscleinvasive bladder cancers, which is concordant with the current medical literature. Our study also determined that frequency of haematuria was more statistically significant in muscle-invasive tumours compared with that in superficial tumours. According to the data from the USA, men were 2.5 times more likely to be diagnosed with bladder cancer than women. In Turkey, this ratio is 7:1. Bladder cancer is a disease of old age; the average age of diagnosis is 72 years (18). Our study sample comprised 276 men (83.4\%) and 55 women (16.6\%), which is concordant with the national data.

The average age of patients in our group was 64 , which is a bit lower than the national average. The increase of industrial and environmental carcinogens in the last 50 years is to blame for the decrease in the age of bladder cancer patients. Since 
our patients were of advanced age, comorbid pathologies such as hypertension, diabetes mellitus, ischaemic heart disease, chronic bladder failure) and chronic obstructive pulmonary disease were also present. Previous studies have shown that delay in bladder cancer diagnosis in men and women (especially delays $>6$ months after the diagnosis and $>12$ weeks from the diagnosis to cystectomy) negatively affects survival rates $(19,20)$. In recent studies, women were found to be at a higher risk of delayed diagnosis and presented with more advanced sickness compared with that in men (21). A study in women with bladder cancer revealed that urological consultations were not performed immediately upon complaints of symptoms and that these patients were given three or more doses of antibiotics first (22). The period between initial haematuria and bladder cancer diagnosis was statistically significant in women (23).

Our study differed from other similar studies in showing that haematuria duration was significantly higher in men than in women $(p=0.035)$. This may be due to the lower ratio of female patients nationally. There were 276 (83.4\%) men and $55(16.6 \%)$ women, and this was concordant with the national data.

Age and haematuria duration were negatively significant when patients were analysed according to age $(p=0.024)$.

When comparing patients with haematuria and those without it, the number tumours $>3 \mathrm{~cm}$ in size were significantly higher in the former $(p=0.045)$. When patients with haematuria were divided into subgroups according to tumour diameter, stage and quantity, patients with Ta tumours were found to have statistically lower frequency of haematuria than in those with T2 tumours. Studies that have examined microvascular invasion of advanced stage tumours support our findings.

A significant association was seen between haematuria duration of 6-12 months and grade 1 toumors. We found that in such patients with long-term macroscopic haematuria, since there was no physical discomfort, diagnoses could not be established either clinically or radiologically. That leads us to stress the importance of cystoscopic controls for definite diagnosis.

The correlation between cigarettes and bladder tumours is clear in medical literature and The European Association of Urology (EAU) guidelines. The emergence of a positive relationship of haematuria duration with cigarette usage (packs/year) in our study supports these findings. Additionally, cigarette use and recurrence of bladder tumours in the macroscopic haematuria group showed a negative correlation. In other words, patients with macroscopic haematuria who also smoked had early recurrences.

We found no relationship between haematuria duration and number of recurrences and tumour prognosis. Age and BMI were also not significantly associated with progression and recurrence in patients with haematuria.

Among the different groups based on the haematuria duration, no significant differences were observed regarding the use of preoperative anticoagulants and the tumour stage, grade, burden and diameter, number of recurrences, time until recurrence, time until progression. Findings from previous studies differ from ours in this respect.
The incidence of haematuria in patients treated with fibrinolytic agents varies between $20 \%$ and 30\% (24). Despite these findings, it is unclear if anticoagulant-related haematuria causes asymptomatic genitourinary lesions to be detected earlier (25).

Generally, the degree of haematuria depends on the amounts and dosage of anticoagulants used. Nevertheless, haematuria may be the only uropathological finding. Therefore, some authors recommend a complete urological evaluation of all patients with non-traumatic anticoagulant-related haematuria. Furthermore, it has also been seen that $30 \%$ of patients receiving anticoagulants were diagnosed with a malignancy (25).

Some authors have claimed that bleeding episodes in patients receiving anticoagulant therapy correlate with PT (26).

One particular study showed that patients attending the emergency room with first time macroscopic haematuria and those on anticoagulant therapy, newly diagnosed with bladder cancers, have a high probability of early-stage and low-grade bladder cancer (27).

The reason that no statistically significant differences in stage and grade, and anticoagulant use emerged in our study may be attributed to the small sample size. Although patients with haematuria numbered 270, only 27 received anticoagulants. This number may be insufficient for meaningful statistical analysis.

\section{Study Limitations}

Although we are proud of the contribution we have made, we feel that this is a topic that should be made the subject of a larger sample size and longer-term prospective randomised studies than ours.

\section{Conclusion}

Haematuria is a severe symptom that motivates patients to seek early treatment. Macroscopic haematuria usually signals an underlying pathology in that $50 \%$ of patients with the symptom are diagnosed with significant urogenital system pathology. Therefore, advanced evaluation is needed to determine its underlying cause. Our study intended to emphasise the most widely known diagnostic symptom of bladder cancers, which is macroscopic haematuria, and to assess it according to its duration and frequency.

Our study determined that frequency of haematuria was significantly higher in muscle-invasive tumours than in superficial tumours. In patients with haematuria, the incidence of tumours $>3 \mathrm{~cm}$ was higher than in those without it.

A positive correlation was determined between haematuria duration and cigarette packs smoked per year. A negative correlation was determined between cigarette use by patients with haematuria and time until recurrence.

Age and haematuria duration were found to be negatively significant. In males, the mean haematuria duration was significantly higher than in females.

We could not find a significant relation between haematuria duration and number of recurrences and tumour prognosis. Also, in patients with haematuria, age and BMI were not significantly associated with tumour progression and recurrence. 
In patients with pre-operative anticoagulant usage, there was no meaningful correlation between haematuria duration and tumour stage, grade, burden, diameter, recurrence and progression.

In contemporary medical literature, a detailed analysis of macroscopic haematuria is lacking, especially regarding its relationship with features of bladder cancers, such as stage, size, recurrence and prognosis. With this study, we hope to contribute to this vital area.

\section{Acknowledgements}

Publication: The results of the study were not published in full or in part in form of abstracts.

Contribution: There is not any contributors who may not be listed as authors.

Conflict of Interest: No conflict of interest was declared by the authors.

Financial Disclosure: The authors declared that this study received no financial support.

Ethics

Ethics Committee Approval: The study protocol was approved by the Cerrahpaşa Medical Faculty Ethics Committee (83045809/604.01/02-218946).

Informed Consent: The consent form was filled in by all participants and their families.

Peer-review: Externally peer-reviewed.

\section{Authorship Contributions}

Critical Review: C.Y., Supervision: S.L.K., Concept: A.H.Y., Design: A.T.A., Data Collection or Processing: E.A., Analysis or Interpretation: G.B., Literature Search: G.B., Writing: H.A.

\section{References}

1. Jemal A, Siegel R, Ward E, et al. Cancer statistics, 2006. CA Cancer J Clin 2006;56:106-130.

2. Zorlu F, Eser S, Fidaner C. İmir ilinde ürogenital kanserlerin insidans hızları. Üroonkoloji Bülteni 2004;1:2-9.

3. Jacobs BL, Lee CT, Montie JE. Bladder cancer in 2010: how far have we come? CA Cancer J Clin 2010;60:244-272.

4. Jones JS. Non-muscle-invasive bladder cancer (Ta, T1, and $\mathrm{CIS}$ ). Campbell-Walsh Urology 2007;3:9.

5. Jichlinski P, Leisinger HJ. Fluorescence cystoscopy in the management of bladder cancer: a help for the urologist. Urol Int 2005;74:97-101.

6. Bubendorf L. Multiprobe fluorescence in situ hybridization (UroVysion) for the detection of urothelial carcinoma-FISHing for the right catch. Acta Cytol 2011;55:113-119.

7. Khadra M, Pickard RS, Charlton $\mathrm{M}$, et al. A prospective analysis of 1,930 patients with hematuria to evaluate current diagnostic practice. J Urol 2000;163:524-527.

8. Mariani A, Mariani MC, Macchioni C, et al. The significance of adult hematuria: 1,000 hematuria evaluations including a risk-benefit and cost-effectiveness analysis. J Urol 1989;141:350-355.
9. Grossfeld GD, Litwin MS, Wolf JS Jr, et al. Evaluation of asymptomatic microscopic hematuria in adults: the American Urological Association best practice policy-part II: patient evaluation, cytology, voided markers, imaging, cystoscopy, nephrology evaluation, and followup. Urology 2001;57:604-610.

10. Cohen RA, Brown RS. Clinical practice. Microscopic hematuria. N Engl J Med 2003;348:2330-2338.

11. Mohr DN, Offord KP, Owen RA, Melton LJ. Asymptomatic microhematuria and urologic disease: a population-based study. JAMA 1986;256:224-229.

12. Edwards TJ, Dickinson AJ, Natale $S$, et al. A prospective analysis of the diagnostic yield resulting from the attendance of 4020 patients at a protocol-driven haematuria clinic. BJU Int 2006;97:301-305; discussion 305.

13. Gandrup KL, Løgager VB, Bretlau T, et al. Diagnosis of bladder tumors in patients with macroscopic haematuria: a prospective comparison of split-bolus computed tomography urography, magnetic resonance urography and flexible cystoscopy. Scand J Urol 2015;49:224-229.

14. Sutton JM. Evaluation of hematuria in adults. JAMA 1990;263:2475-2480

15. Gore JL, Lai J, Setodji CM, et al. Mortality increases when radical cystectomy is delayed more than 12 weeks: results from a Surveillance, Epidemiology, and End Results-Medicare analysis. Cancer 2009;115:988-996.

16. Wallace D, Bryan RT, Dunn JA, et al. Delay and survival in bladder cancer. BJU Int 2002;89:868-878.

17. Hollenbeck BK, Dunn RL, Ye Z, et al. Delays in diagnosis and bladder cancer mortality. Cancer 2010;116:5235-5242.

18. Özen H, Turkeri L. Uroonkoloji Kitabi. 2007;151-257.

19. Sanchez-Ortiz RF, Huang WC, Mick R, et al. An interval longer than 12 weeks between the diagnosis of muscle invasion and cystectomy is associated with worse outcome in bladder carcinoma. J Urol 2003;169:110-115; discussion 115

20. Lee CT, Madii R, Daignault S, et al. Cystectomy delay more than 3 months from initial bladder cancer diagnosis results in decreased disease specific and overall survival. J Urol 2006;175:1262-1267; discussion 1267

21. Mallin K, David KA, Carroll PR, et al. Transitional cell carcinoma of the bladder: racial and gender disparities in survival (1993 to 2002), stage and grade (1993 to 2007). J Urol 2011;185:1631-1636.

22. Henning $A$, Wehrberger $M$, Madersbacher $S$, et al. Do differences in clinical symptoms and referral patterns contribute to the gender gap in bladder cancer? BJU Int 2013;112:68-73.

23. Cohn JA, Vekhter B, Lyttle C, et al. Sex disparities in diagnosis of bladder cancer after initial presentation with hematuria: A nationwide claims-based investigation. Cancer 2014;120:555-561.

24. Antoniewicz AA, Zapała L, Poletajew S, Borowka A. Macroscopic hematuria-a leading urological problem in patients on anticoagulant therapy: is the common diagnostic standard still advisable? ISRN Urol 2012;2012:710734.

25. Van Savage JG, Fried FA. Anticoagulant associated hematuria: a prospective study. J Urol 1995;153:1594-1596.

26. Coon WW, Willis PW 3rd. Hemorrhagic complications of anticoagulant therapy. Arch Intern Med 1974;133:386-392.

27. Moschini M, Karnes RJ, Suardi N, et al. Potential Effect of Antiplatelet and Anticoagulant Therapy on the Timing of the Diagnosis of Bladder Cancer. Clin Genitourin Cancer 2016;14:e245-e250. 